SISSA-ISAS 144/95/EP

IFT-P057/95

\title{
Toda lattice realization of integrable hierarchies
}

\author{
L.Bonora \\ International School for Advanced Studies (SISSA/ISAS) \\ Via Beirut 2, 34014 Trieste, Italy, and \\ INFN, Sezione di Trieste. \\ C.P.Constantinidis \\ Instituto de Física Teórica - UNESP \\ Rua Pamplona 145, 01405 São Paulo, Brasil \\ E.Vinteler \\ International School for Advanced Studies (SISSA/ISAS) \\ Via Beirut 2, 34014 Trieste, Italy
}

\begin{abstract}
We present a new realization of scalar integrable hierarchies in terms of the Toda lattice hierarchy. In other words, we show on a large number of examples that an integrable hierarchy, defined by a pseudodifferential Lax operator, can be embedded in the Toda lattice hierarchy. Such a realization in terms the Toda lattice hierarchy seems to be as general as the Drinfeld-Sokolov realization.
\end{abstract}




\section{Introduction}

Scalar integrable hierarchies can be introduced in terms of (pseudo)differential operators by means of a formalism first introduced by Gelfand and Dickey (see [1]). This is the most 'disembodied' form in which such hierarchies can appear, and it can be taken as a reference form. One can then consider realizations of these hierarchies in physical systems. A comprehensive realization is the one studied by Drinfeld and Sokolov in terms of linear systems defined on Lie algebras, [2]; let us refer to it as the Drinfeld-Sokolov realization (DSR). In this letter we present a new general realization of integrable hierarchies in terms of the Toda lattice hierarchy (TLH). We call it Toda lattice realization (TLR), and it looks as general as the DSR. While the DSR is contiguous to (reduced) WZNW models and Toda field theories in 2D, the TLR is inspired by matrix models, see [3], 价].

The letter is organized as follows. In section 2 we introduce the TLR. We do not give a general proof of it, but in section 3 we verify it on a large number of examples among KP, n-KdV and other classes of hierarchies. Section 5 is devoted to some comments.

\section{The Toda lattice realization of integrable hierar- chies.}

In the Gelfand-Dickey (GD) formalism an integrable hierarchy can be entirely specified in terms of the Lax operator

$$
L=\partial^{N}+N a_{1} \partial^{N-2}+N a_{2} \partial^{N-3}+\ldots+N a_{N-1}+N a_{N} \partial^{-1}+\ldots
$$

where $\partial=\frac{\partial}{\partial x}$. The operator $L$ may be purely differential, in which case $a_{k}=0$ for $k \geq N$, and we get the $N-\mathrm{KdV}$ hierarchy. The fields $a_{k}$ may be either elementary or composite of more elementary fields, as in the case of the $(N, M)-\mathrm{KdV}$ hierarchies studied in [5], [6], [7]. If the hierarchy is integrable, the flows are given by

$$
\frac{\partial L}{\partial t_{k}}=\left[\left(L^{k / N}\right)_{+}, L\right]
$$

where the subscript + denotes the differential part of a pseudodifferential operator, $t_{1}$ is identified with $x$ and $k$ spans a specific subset of the positive integers.

The Toda lattice hierarchy is defined in terms of a semi-infinite Jacobi matrix $\hat{Q}$. We parametrize it as follows

$$
\hat{Q}=\sum_{j=0}^{\infty}\left(E_{j, j+1}+\sum_{l=0}^{\infty} \hat{a}_{l}(j) E_{j+l, j}\right), \quad\left(E_{j, m}\right)_{k, l}=\delta_{j, k} \delta_{m, l}
$$

and consider $\hat{a}_{l}$ as fields defined on a lattice. The flows are given by

$$
\frac{\partial \hat{Q}}{\partial t_{k}}=\left[\left(\hat{Q}^{k}\right)_{+}, \hat{Q}\right], \quad k=1,2, \ldots
$$

${ }^{*}$ In this paper we limit ourselves to a simple version of the TLH, in which only one matrix $\hat{Q}$ and one set of parameters intervene, instead of two or more [10], [3] 
where the subscript + denotes the upper triangular part of a matrix, including the main diagonal. (2.4) represents a hierarchy of differential-difference equations for the fields $a_{l}$. In particular the first flows are

$$
\hat{a}_{l}(j)^{\prime}=\hat{a}_{l+1}(j+1)-\hat{a}_{l+1}(j)+\hat{a}_{l}(j)\left(\hat{a}_{0}(j)-\hat{a}_{0}(j-l)\right)
$$

where we have adopted the notation $\frac{\partial}{\partial t_{1}} f \equiv f^{\prime} \equiv \partial f$, for any function $f$. The parameter $t_{k}$ of the TLH will be identified later on with the corresponding parameter $t_{k}$ in (2.2) whenever the latter exists; therefore, in particular, $t_{1}$ will be identified with $x$.

Next, integrability permits us to introduce the function $\hat{F}(n, t)$ (the free energy in matrix models) via

$$
\frac{\partial^{2}}{\partial t_{k} \partial t_{l}} \hat{F}(n, t)=\operatorname{Tr}\left(\left[\hat{Q}_{+}^{k}, \hat{Q}^{l}\right]\right)
$$

where $\operatorname{Tr}(X)$ denotes the finite trace $\sum_{j=0}^{n-1} X_{j, j}$. In particular (2.6) leads to

$$
\frac{\partial^{2}}{\partial t_{1}^{2}} \hat{F}(n, t)=\hat{a}_{1}(n)
$$

It is clear that by means of (2.4) we can compute the derivatives of any order of $\hat{F}$ in terms of the entries of $\hat{Q}$. In general we will denote by $\hat{F}_{k_{1}, \ldots, k_{s}}$ the derivative of $\hat{F}$ with respect to $t_{k_{1}}, \ldots, t_{k_{s}}$.

Next we introduce the operator $D_{0}$, defined by its action on any discrete function $f(n)$

$$
\left(D_{0} f\right)(n)=f(n+1)
$$

For later use we remark that, if $f_{0}=0$, the operation $\operatorname{Tr}$ is the inverse of the operation $D_{0}-1$. We will also use the notation $e^{\partial_{0}}$ instead of $D_{0}$, with the following difference: $D_{0}$ is meant to be applied to the nearest right neighbour, while $e^{\partial_{0}}$ acts on whatever is on its right. Now we can equivalently represent the matrix $\hat{Q}$ by the following operator

$$
\hat{Q}(j)=e^{\partial_{0}}+\sum_{l=0}^{\infty} \hat{a}_{l}(j) e^{-l \partial_{0}}
$$

The contact between (2.8) and (2.3) is made by acting with the former on a discrete function $\xi(j)$; then $\hat{Q}(j) \xi(j)$ is the same as the $j$-th component of $\hat{Q} \xi$, where $\xi$ is a column vector with components $\xi(0), \xi(1), \ldots$. We will generally drop the dependence on $j$ in (2.8) and merge the two symbols.

After this short introduction to the GD formalism and the Toda lattice hierarchy, let us come to the presentation of the TLR of the integrable hierarchy defined by the Lax operator (2.1), i.e. to the problem of embedding the latter into the TLH. The prescription consists of several steps.

Step 1. In $\hat{Q}$ we set $\hat{a}_{0}=0$ and replace the first flows (2.5) with

$$
D_{0} \hat{a}_{1}=\hat{a}_{1}, \quad D_{0} \hat{a}_{i}=\hat{a}_{i}+\hat{a}_{i-1}^{\prime}, \quad i=2,3, \ldots
$$

Step 2. We compute

$$
\frac{\partial \hat{a}_{1}}{\partial t_{k}}=\partial \operatorname{Tr}\left(\left[\hat{Q}_{+}, \hat{Q}^{k}\right]\right) \equiv \partial \hat{F}_{1, k}
$$


The right hand side will be a polynomial of the fields $\hat{a}_{k}$ to which monomials of $D_{0}$ and $D_{0}^{-1}$ are applied. Next we substitute the first flows (2.9) to eliminate the presence of $D_{0}$. Examples:

$$
\begin{aligned}
& \hat{F}_{1,1}=\hat{a}_{1}, \\
& \hat{F}_{1,2}=\left(D_{0}+1\right) \hat{a}_{2}=2 \hat{a}_{2}+\hat{a}_{1}^{\prime}, \\
& \hat{F}_{1,3}=\left(D_{0}^{2}+D_{0}+1\right) \hat{a}_{3}+D_{0} \hat{a}_{1} \hat{a}_{1}+\hat{a}_{1} \hat{a}_{1}+\hat{a}_{1} D_{0}^{-1} \hat{a}_{1}=3 \hat{a}_{3}+3 \hat{a}_{2}^{\prime}+\hat{a}_{1}^{\prime \prime}+3 \hat{a}_{1}^{2}
\end{aligned}
$$

and so on.

Next we recall that

$$
\hat{F}_{1, k}=\frac{\partial^{2}}{\partial t_{k} \partial t_{1}} \hat{F}
$$

Using this and (2.11), we can recursively write all the derivatives of $\hat{a}_{l}$ with respect to the couplings $t_{k}$ (and in particular the flows) in terms of derivatives of $\hat{F}$, which, in turn, can be expressed as functions of the entries of $\hat{Q}$. Example:

$$
\frac{\partial}{\partial t_{k}} \hat{a}_{2}=\frac{1}{2} \partial \operatorname{Tr}\left(\left[\hat{Q}_{+}^{2}, \hat{Q}^{k}\right]\right)-\frac{1}{2} \frac{\partial}{\partial t_{k}} \hat{a}_{1}^{\prime}
$$

In general we will need all $\hat{F}_{k_{1}, \ldots, k_{n}}$. Here are some general formulas. Let us introduce the symbols $\hat{A}_{j}^{[k]}$ as follows

$$
\hat{Q}^{k}=e^{k \partial}+k a_{1} e^{(k-2) \partial}+\hat{A}_{2}^{[k]} e^{(k-3) \partial}+\hat{A}_{3}^{[k]} e^{(k-4) \partial}+\hat{A}_{4}^{[k]} e^{(k-5) \partial}+\ldots
$$

The explicit form of the first few is:

$$
\begin{aligned}
\hat{A}_{2}^{[k]}= & \left(\begin{array}{c}
k \\
2
\end{array}\right) a_{1}^{\prime}+k \hat{a}_{2} \\
\hat{A}_{3}^{[k]}= & \left(\begin{array}{c}
k \\
3
\end{array}\right) a_{1}^{\prime \prime}+\left(\begin{array}{c}
k \\
2
\end{array}\right) \hat{a}_{2}^{\prime}+k \hat{a}_{3}+\left(\begin{array}{c}
k \\
2
\end{array}\right) a_{1}^{2} \\
\hat{A}_{4}^{[k]}= & \left(\begin{array}{c}
k \\
4
\end{array}\right) a_{1}^{\prime \prime \prime}+\left(\begin{array}{c}
k \\
3
\end{array}\right) \hat{a}_{2}^{\prime \prime}+\left(\begin{array}{c}
k \\
2
\end{array}\right) \hat{a}_{3}^{\prime}+k \hat{a}_{4}+\left(3\left(\begin{array}{c}
k \\
3
\end{array}\right)-\left(\begin{array}{c}
k \\
2
\end{array}\right)\right) a_{1} a_{1}^{\prime}+2\left(\begin{array}{c}
k \\
2
\end{array}\right) a_{1} \hat{a}_{2} \\
\hat{A}_{5}^{[k]}= & \left(\begin{array}{c}
k \\
5
\end{array}\right) a_{1}^{(4)}+\left(\begin{array}{c}
k \\
4
\end{array}\right) \hat{a}_{2}^{\prime \prime \prime}+\left(\begin{array}{c}
k \\
3
\end{array}\right) \hat{a}_{3}^{\prime \prime}+\left(\begin{array}{c}
k \\
2
\end{array}\right) \hat{a}_{4}^{\prime}+k \hat{a}_{5}+\left(\begin{array}{c}
k \\
2
\end{array}\right) \hat{a}_{2} \hat{a}_{2}+2\left(\begin{array}{c}
k \\
2
\end{array}\right) a_{1} \hat{a}_{3} \\
& +\left(\begin{array}{l}
k \\
3
\end{array}\right) a_{1}^{3}+\left(3\left(\begin{array}{c}
k \\
4
\end{array}\right)-\left(\begin{array}{c}
k \\
3
\end{array}\right)\right) a_{1}^{\prime} a_{1}^{\prime}+\left(4\left(\begin{array}{c}
k \\
4
\end{array}\right)-2\left(\begin{array}{c}
k \\
3
\end{array}\right)+\left(\begin{array}{c}
k \\
2
\end{array}\right)\right) a_{1} a_{1}^{\prime \prime}+ \\
& \left(3\left(\begin{array}{c}
k \\
3
\end{array}\right)-\left(\begin{array}{c}
k \\
2
\end{array}\right)\right) a_{1} \hat{a}_{2}^{\prime}+\left(3\left(\begin{array}{l}
k \\
3
\end{array}\right)-2\left(\begin{array}{c}
k \\
2
\end{array}\right)\right) \hat{a}_{2} a_{1}^{\prime}
\end{aligned}
$$

and so on. In terms of these coefficients we can compute all the derivatives of $\hat{F}$. For example

$$
\begin{aligned}
\hat{F}_{1, k} & =\hat{A}_{k}^{[k]} \\
\hat{F}_{2, k} & =\left(D_{0}+1\right) \hat{A}_{k+1}^{[k]} \\
\hat{F}_{3, k} & =\left(D_{0}^{2}+D_{0}+1\right) \hat{A}_{k+2}^{[k]}+3 a_{1} \hat{A}_{k}^{[k]} \\
\hat{F}_{4, k} & =\left(D_{0}^{3}+D_{0}^{2}+D_{0}+1\right) \hat{A}_{k+3}^{[k]}+4 a_{1}\left(D_{0}+1\right) \hat{A}_{k+1}^{[k]}+a_{2}^{(4)} \hat{A}_{k}^{[k]}
\end{aligned}
$$


This procedure allows us to compute all the derivatives of the fields $\hat{a}_{l}$ in terms of the same fields and their derivatives with respect to $x \equiv t_{1}$ - therefore, in particular, the flows.

So far all our moves have been completely general (except for setting $\hat{a}_{0}=0$, but see the comment at the end of this section). The next step is instead a 'gauge choice', that is we make a particular choice for the matrix $\hat{Q}$. The word 'gauge' is not merely colorful. In fact gauge transformations play here a role analogous to gauge transformations in [2]. The relevant gauge transformations in the present case are defined by $\hat{Q} \rightarrow G_{-} \hat{Q} G_{-}^{-1}$, where $G_{-}$is a strictly lower triangular semi-infinite matrix.

Step 3. We fix the gauge by imposing the condition

$$
\hat{Q}^{N}=e^{N \partial_{0}}+\sum_{l=1} a_{l} e^{(N-1-l) \partial_{0}}
$$

where the $a_{l}$ are the same as in eq.(2.1). The matrix $\hat{Q}$ that satisfies such condition will be referred to as $\bar{Q}$. It is clear that $\bar{Q}^{N}$ exactly mimics the Lax operator $L$. The condition (2.15) recursively determines $\hat{a}_{k}$ in terms of the fields $a_{l}$ that appear in $L$.

$$
\hat{a}_{k}=\bar{a}_{k} \equiv P_{k}\left(a_{l}\right)
$$

where $P_{k}$ are differential polynomials of $a_{l}$. In particular we always have $\hat{a}_{1}=\bar{a}_{1} \equiv a_{1}$.

Step 4. Then we evaluate both sides of the flows found in Step 2 at $\hat{a}_{k}=\bar{a}_{k}$. The order here is crucial. The gauge fixing of the flows must be the last operation.

Now we claim:

Claim. The flows obtained in this way coincide with the flows (2.7) for corresponding couplings.

We will substantiate this claim with a large number of examples in the next section.

It is perhaps useful to summarize our method: start from the TLH flows, use the first flows (2.9) and impose the relevant gauge fixing; the resulting flows are the desired differential integrable flows.

We would like to end this section with a remark concerning the restriction $\hat{a}_{0}=0$ we imposed at the very beginning. This can be avoided at the price of working with very encumbering formulas. One can keep $\hat{a}_{0} \neq 0$ provided one uses the first flows $(2.5)$ instead of (2.9) in Step 1. In this way it is possible, in general, to eliminate $D_{0}$ in the flows only when it acts over $\hat{a}_{l}, l \neq 0$ (see the last section for an additional comment on this point). We obtain in this way the same equations as above with the addition of terms involving $\hat{a}_{0}$. We can suppress all these additional terms at the end (Step 5) by imposing $\hat{a}_{0}=0$ as part of the gauge choice. The final result is of course the same as before. This justifies our having imposed $\hat{a}_{0}=0$ from the very beginning.

\section{Examples.}

In this section we present a large number of examples in support of the claim of the previous section. Of course for obvious reasons of space we can explicitly exhibit a few cases only, and for each case only a few flows among those we have checked. 


\section{The KP hierarchy}

The KP case corresponds to $n=1$ in (2.1). Therefore there is no gauge fixing: $\hat{a}_{l}=a_{l}$. The flows obtained with our method are simply those in Step 3. Examples:

$$
\begin{array}{lll}
\frac{\partial \hat{a}_{1}}{\partial t_{2}}=\left(2 \hat{a}_{2}+\hat{a}_{1}^{\prime}\right)^{\prime}, & \frac{\partial \hat{a}_{1}}{\partial t_{3}}=\left(3 \hat{a}_{3}+3 \hat{a}_{2}^{\prime}+\hat{a}_{1}^{\prime \prime}+3 \hat{a}_{1}^{2}\right)^{\prime} \\
\frac{\partial \hat{a}_{2}}{\partial t_{2}}=\left(2 \hat{a}_{3}+\hat{a}_{2}^{\prime}+\hat{a}_{1}^{2}\right)^{\prime}, & \frac{\partial \hat{a}_{2}}{\partial t_{3}}=\left(3 \hat{a}_{4}+3 \hat{a}_{3}^{\prime}+\hat{a}_{2}^{\prime \prime}+6 \hat{a}_{1} \hat{a}_{2}\right)^{\prime}
\end{array}
$$

and so on. Setting $\hat{a}_{l}=a_{l}$, these are exactly the KP flows.

\section{The $N$-KdV hierarchy case}

In 44 we have explicitly shown that our claim is true for the $3-\mathrm{KdV}$ hierarchy. In this section we generalize that result. To start with we pick a generic $N$. The relevant differential operator is

$$
L=D^{N}+N a_{1} D^{N-2}+N a_{2} D^{N-3}+\ldots+N a_{N-1}
$$

We also write

$$
L^{k / N}=D^{k}+k a_{1} D^{k-2}+b_{2}^{[k]} D^{k-3}+\ldots+b_{j-1}^{[k]} D^{k-j}+\ldots
$$

The coefficients $b_{j}^{[k]}$ are differential polynomials in $a_{l}, l=1, \ldots, a_{N-2}$.

Working out the commutator in relation (2.2), we can write down the general formula for arbitrary flow $t_{m}$ :

$$
\begin{aligned}
\frac{\partial a_{j-1}}{\partial t_{m}} & =\sum_{k=0}^{m-1}\left(\begin{array}{c}
m \\
k
\end{array}\right) a_{j+k-1}^{(m-k)}-\sum_{k=0}^{m-2} \frac{1}{N}\left(\begin{array}{c}
N \\
j+k
\end{array}\right)\left(b_{m-k-1}^{(m)}\right)^{(j+k)}+ \\
& +\sum_{k=0}^{m-3}\left(\left(\begin{array}{c}
m-2 \\
k
\end{array}\right) b_{k-1}^{(m)} a_{j-k-1}^{(m-2-k)}-\sum_{l=0}^{m-2}\left(\begin{array}{c}
N-j-k \\
l-k
\end{array}\right) a_{k-1}\left(b_{m-l-1}^{(m)}\right)^{(l-k)}\right) \\
& -\sum_{k=2}^{j-1} \sum_{l=0}^{m-2}\left(\begin{array}{c}
N-k \\
j-k+l
\end{array}\right) a_{k-1}\left(b_{m-l-1}^{(m)}\right)^{(j-k+l)}
\end{aligned}
$$

Now let us pass to the TLR of this hierarchy. We recall eqs.(2.13) and (2.14). We fix the gauge by imposing $\hat{A}_{j}^{[N]}=N a_{j}$. We solve the equations for $\hat{a}_{j}$ in terms of $a_{j}$ and obtain $\bar{a}_{j}$. Next we insert back the result in the formulas of the coefficients $\hat{A}_{j}^{[k]}$ so that they become functions of $a_{j}$. We call the result $\bar{A}_{j}^{[k]}$. Examples:

$$
\begin{aligned}
\bar{A}_{2}^{[k]} / k= & a_{2}-\frac{N-k}{2} a_{1}^{\prime} \\
\bar{A}_{3}^{[k]} / k= & a_{3}-\frac{N-k}{2} a_{2}^{\prime}+\frac{(N-2 k+3)(N-k)}{12} a_{1}^{\prime \prime}-\frac{N-k}{2} a_{1}^{2} \\
\bar{A}_{4}^{[k]} / k= & a_{4}-\frac{N-k}{2} a_{3}^{\prime}+\frac{(N-2 k+3)(N-k)}{12} a_{2}^{\prime \prime}-\frac{(N-k+2)(k-2)(N-k)}{24} a_{1}^{\prime \prime \prime} \\
& -(N-k) a_{1} a_{2}+\frac{(N-k+2)(N-k)}{2} a_{1} a_{1}^{\prime}
\end{aligned}
$$


Then, using our recipe, we obtain

$$
\begin{aligned}
\partial^{-1} \frac{\partial a_{1}}{\partial t_{k}} & =\left.\frac{\partial^{2} F}{\partial t_{1} \partial t_{k}}\right|_{\hat{a}=\bar{a}}=\bar{A}_{k}^{[k]} \\
\partial^{-1} \frac{\partial a_{2}}{\partial t_{k}} & =\left.\left(\frac{1}{2} \frac{\partial^{2} F}{\partial t_{2} \partial t_{k}}\right)\right|_{\hat{a}=\bar{a}}+\frac{N-2}{2} \frac{\partial a_{1}}{\partial t_{k}}=\bar{A}_{k+1}^{[k]}+\frac{N-1}{2}\left(\bar{A}_{k}^{[k]}\right)^{\prime} \\
\partial^{-1} \frac{\partial a_{3}}{\partial t_{k}} & =\left.\left(\frac{1}{3} \frac{\partial^{2} F}{\partial t_{3} \partial t_{k}}\right)\right|_{\hat{a}=\bar{a}}+\frac{N-3}{2} \frac{\partial a_{2}}{\partial t_{k}}-\frac{(N-3)^{2}}{12} \frac{\partial a_{1}^{\prime}}{\partial t_{k}}+(N-3) \partial^{-1}\left(a_{1} \frac{\partial a_{1}}{\partial t_{k}}\right) \\
& =\bar{A}_{k+2}^{[k]}+\frac{N-1}{2}\left(\bar{A}_{k+1}^{[k]}\right)^{\prime}+\frac{(N-1)(N-2)}{6}\left(\bar{A}_{k}^{[k]}\right)^{\prime \prime}+a_{1} \bar{A}_{k}^{[k]}+(N-3) \partial^{-1}\left(a_{1}\left(\bar{A}_{k}^{[k]}\right)^{\prime}\right) \\
\partial^{-1} \frac{\partial a_{4}}{\partial t_{k}} & =\left.\left(\frac{1}{4} \frac{\partial^{2} F}{\partial t_{4} \partial t_{k}}+\frac{N-4}{2} \frac{\partial a_{3}}{\partial t_{k}}\right)\right|_{\hat{a}=\bar{a}}-\frac{(N-4)(N-5)}{12} \frac{\partial a_{2}^{\prime}}{\partial t_{k}}+(N-4) \partial^{-1} \frac{\partial\left(a_{1} a_{2}\right)}{\partial t_{k}} \\
& -\frac{(N-4)(N-2)}{2}\left(\frac{1}{6} \frac{\partial a_{1}^{\prime \prime}}{\partial t_{k}}+a_{1} \frac{\partial a_{1}}{\partial t_{k}}\right)=\bar{A}_{k+3}^{[k]}+\frac{N-1}{2}\left(\bar{A}_{k+2}^{[k]}\right)^{\prime} \\
& +\frac{(3 N-11)(N-1)(N-2)}{24}\left(\bar{A}_{k}^{[k]}\right)^{\prime \prime \prime}-\frac{(N-2)(N-4)}{2} a_{1}\left(\bar{A}_{k}^{[k]}\right)^{\prime}+ \\
& +\frac{(5 N-16)(N-1)}{12}\left(\bar{A}_{k+1}^{[k]}\right)^{\prime \prime}+(N-4) \partial^{-1}\left(a_{1}\left(\bar{A}_{k+1}^{[k]}\right)^{\prime}+\frac{N-1}{2}\left(a_{1}\left(\bar{A}_{k}^{[k]}\right)^{\prime \prime}+a_{2}\left(\hat{A}_{k}^{[k]}\right)^{\prime}\right)\right.
\end{aligned}
$$

and so on, where $\hat{a}=\bar{a}$ denotes gauge fixing.

We give a few concrete examples of the second and third flows:

$$
\begin{aligned}
\partial^{-1} \frac{\partial a_{1}}{\partial t_{2}} & =2 a_{2}-(N-2) a_{1}^{\prime} \\
\partial^{-1} \frac{\partial a_{2}}{\partial t_{2}}= & 2 a_{3}+a_{2}^{\prime}-\frac{(N-1)(N-2)}{3} a_{1}^{\prime \prime}-(N-2) a_{1}^{2} \\
\frac{\partial a_{3}}{\partial t_{2}}= & 2 a_{4}^{\prime}+a_{3}^{\prime \prime}-\frac{(N-1)(N-2)(N-3)}{12} a_{1}^{(4)}-(N-2)(N-3) a_{1} a_{1}^{\prime \prime}-2(N-3) a_{2} a_{1}^{\prime} \\
\partial^{-1} \frac{\partial a_{1}}{\partial t_{3}}= & 3 a_{3}-\frac{3}{2}(N-3) a_{2}^{\prime}+\frac{(N-3)^{2}}{4} a_{1}^{\prime \prime}-\frac{3}{2}(N-3) a_{1}^{2} \\
\partial^{-1} \frac{\partial a_{2}}{\partial t_{3}}= & 3 a_{4}+3 a_{3}^{\prime}-\frac{N(N-3)}{2} a_{2}^{\prime \prime}+\frac{(N-1)(N-2)(N-3)}{8} a_{1}^{\prime \prime \prime}-3(N-3) a_{1} a_{2} \\
\frac{\partial a_{3}}{\partial t_{3}}= & 3 a_{5}^{\prime}+3 a_{4}^{\prime \prime}+a_{3}^{\prime \prime \prime}-\frac{3}{N}\left(\begin{array}{c}
N \\
4
\end{array}\right) a_{2}^{(4)}+\frac{3(3 N-7)}{10 N}\left(\begin{array}{c}
N \\
4
\end{array}\right) a_{1}^{(5)}+3 a_{1} a_{3}^{\prime}-3(N-4) a_{1}^{\prime} a_{3} \\
& -3(N-3) a_{2} a_{2}^{\prime}-\frac{3}{2}(N-2)(N-3) a_{1} a_{2}^{\prime \prime}+\frac{3}{2}(N-3) a_{2} a_{1}^{\prime \prime}+\frac{6}{N}\left(\begin{array}{c}
N \\
4
\end{array}\right) a_{1} a_{1}^{\prime \prime \prime}
\end{aligned}
$$

These are flows pertinent to the $N-\mathrm{KdV}$ hierarchy with $N>3$. In general the formulas of the $N-\mathrm{KdV}$ hierarchy and the corresponding formulas obtained with our method coincide since

$$
b_{k}^{[m]}=\bar{A}_{k}^{[m]}
$$

We have checked these identities case by case up to the $5-\mathrm{KdV}$ and for $m \leq 5$. For the dispersionless case we have verified the correspondence up to the $8-\mathrm{KdV}$ flows. 


\section{The DS hierarchies}

Drinfeld and Sokolov, [2], introduced a large set of generalized KdV systems in terms of the pair $\left(G, c_{m}\right)$, where $G$ is a classical Kac-Moody algebra and $c_{m}$ is a vertex of the Dynkin diagram of $G$. From each choice of the pair $\left(G, c_{m}\right)$ they were able to construct a pseudo-differential operator $L$ which give rise to a hierarchy of integrable equations. We have studied all the examples corresponding to the operator $L$ of orders $3,4,5$ and found a complete agreement with our method. For simplicity here we present a few examples of order 4 and 5 , corresponding to the cases with a pseudodifferential Lax operator. The cases with a differential Lax operator are restriction of the $4-$ and $5-\mathrm{KdV}$ hierarchies, and will be omitted.

In each case we give the explicit form of the (pseudo-)differential operator $L$, the gauge-fixed matrix $\bar{Q}$ and the first significant flows:

\section{Order 4.}

Case $B_{2}^{(1)}$ :

$$
\begin{aligned}
c_{0}, c_{1}: L= & D^{4}+2 u_{1} D^{2}+u_{1}^{\prime} D+2\left(u_{0}+u_{1}^{\prime \prime}\right)-D^{-1}\left(u_{0}+u_{1}^{\prime \prime}\right)^{\prime} \\
\hat{Q}= & e^{\partial}+\frac{v_{1}}{4} e^{-\partial}-\frac{v_{1}^{\prime}}{4} e^{-2 \partial}+\left(\frac{1}{4} v_{0}+\frac{v_{1}^{\prime \prime}}{8}-\frac{3}{32} v_{1}^{2}\right) e^{-3 \partial}+ \\
& +\left(\frac{3}{8} v_{1} v_{1}^{\prime}-\frac{1}{2} v_{0}^{\prime}\right) e^{-4 \partial}+\ldots \\
c_{2}: L= & D^{4}+2 u_{1} D^{2}+u_{1}^{\prime} D+u_{0}^{2}-u_{0} D^{-1} u_{0}^{\prime}
\end{aligned}
$$

For $c_{2}$ we have, up to the order $e^{-4 \partial}$, the same expression for $\hat{Q}$ with $v_{0}=u_{0}^{2}$. The first non-trivial flows are:

$$
\begin{aligned}
& \frac{\partial v_{1}}{\partial t_{3}}=-\frac{1}{2} v_{1}^{\prime \prime \prime}-\frac{3}{4} v_{1} v_{1}^{\prime}+3 v_{0}^{\prime} \\
& \frac{\partial v_{0}}{\partial t_{3}}=v_{0}^{\prime \prime \prime}+\frac{3}{4} v_{1} v_{0}^{\prime}
\end{aligned}
$$

where for $c_{0}, c_{1}: v_{1}=2 u_{1}, v_{0}=2\left(u_{0}+u_{1}^{\prime \prime}\right)$ and for $c_{2}: v_{1}=2 u_{1}, v_{0}=u_{0}^{2}$.

Case $D_{3}^{(1)}$ :

$$
\begin{aligned}
c_{0}, c_{1}: \quad L= & D^{4}+2 u_{2} D^{2}+u_{2}^{\prime} D+2 u_{2}^{\prime \prime}+2 u_{1}-D^{-1}\left(u_{1}^{\prime}+u_{2}^{\prime \prime}\right)^{\prime}+\left(D^{-1} u_{0}\right)^{2} \\
\hat{Q}= & e^{\partial}+\frac{u_{2}}{2} e^{-\partial}-\frac{1}{2} u_{2}^{\prime} e^{-2 \partial}+\left(\frac{3}{4} u_{2}^{\prime \prime}-\frac{3}{8} u_{2}^{2}-\frac{1}{2} u_{1}\right) e^{-3 \partial}+ \\
& +\left(\frac{3}{2} u_{2} u_{2}^{\prime}-u_{1}^{\prime}-u_{2}^{\prime \prime \prime}\right) e^{-4 \partial}+\ldots \\
c_{2}, c_{3}: \quad L= & D^{4}+2 u_{2} D^{2}+3 u_{2}^{\prime} D+\left(2 u_{1}+3 u_{2}^{\prime \prime}\right)+\left(u_{1}^{\prime}+u_{2}^{\prime \prime \prime}\right) D^{-1}+u_{0} D^{-1} u_{0} D^{-1} \\
\hat{Q}= & e^{\partial}+\frac{u_{2}}{2} e^{-\partial}+\left(\frac{1}{4} u_{2}^{\prime \prime}-\frac{3}{8} u_{2}^{2}+\frac{1}{2} u_{1}\right) e^{-3 \partial}+ \\
& +\left(\frac{3}{4} u_{2} u_{2}^{\prime}-\frac{1}{2} u_{1}^{\prime}-\frac{1}{4} u_{2}^{\prime \prime \prime}\right) e^{-4 \partial}+\ldots
\end{aligned}
$$

The first non-trivial equations for $c_{0}, c_{1}$ are:

$$
\begin{aligned}
& \frac{\partial u_{1}}{\partial t_{3}}=3 u_{0} u_{0}^{\prime}-\frac{3}{2} u_{2}^{(5)}-2 u_{1}^{\prime \prime \prime}+\frac{3}{2} u_{1}^{\prime} u_{2}+3 u_{2} u_{2}^{\prime \prime \prime}+\frac{9}{2} u_{2}^{\prime} u_{2}^{\prime \prime} \\
& \frac{\partial u_{2}}{\partial t_{3}}=\frac{5}{2} u_{2}^{\prime \prime \prime}-\frac{3}{2} u_{2} u_{2}^{\prime}+3 u_{1}^{\prime}
\end{aligned}
$$


and for $c_{2}, c_{3}$ are

$$
\begin{aligned}
\frac{\partial u_{1}}{\partial t_{3}} & =3 u_{0} u_{0}^{\prime}-\frac{3}{2} u_{2}^{(5)}-2 u_{1}^{\prime \prime \prime}-\frac{3}{2} u_{1}^{\prime} u_{2}+\frac{9}{2} u_{2}^{\prime} u_{2}^{\prime \prime}+3 u_{2} u_{2}^{\prime \prime \prime} \\
\frac{\partial u_{2}}{\partial t_{3}} & =\frac{5}{2} u_{2}^{\prime \prime \prime}-\frac{3}{2} u_{2} u_{2}^{\prime}+3 u_{1}^{\prime}
\end{aligned}
$$

\section{Order 5.}

Case $A_{5}^{(2)}$ :

$$
\begin{aligned}
c_{0}, c_{1}: \quad & L=D^{5}+2 u_{2} D^{3}+2 u_{2}^{\prime} D^{2}+\left(2 u_{1}+4 u_{2}^{\prime \prime}\right) D+D^{-1}\left(2 u_{0}+u_{1}^{\prime \prime}+u_{2}^{\prime \prime \prime}\right) \\
& \hat{Q}=e^{\partial}+\frac{2}{5} u_{2} e^{-\partial}-\frac{2}{5} u_{2}^{\prime} e^{-2 \partial}+\frac{2}{5}\left(u_{1}+2 u_{2}^{\prime \prime}-\frac{4}{5} u_{2}^{2}\right) e^{-3 \partial}+\ldots \\
c_{2}: \quad & L=D^{5}+2\left(v_{0}+u_{1}\right) D^{3}+\left(6 v_{0}^{\prime}+u_{1}^{\prime}\right) D^{2}+\left(6 v_{0}^{\prime \prime}+u_{0}^{2}+4 v_{0} u_{1}\right) D+ \\
& +\left(2 v_{0}^{\prime \prime \prime}-u_{0} u_{0}^{\prime}+4 u_{1} v_{0}^{\prime}+2 v_{0} u_{1}^{\prime}\right)+u_{0} D^{-1}\left(u_{0}^{\prime \prime}+2 u_{0} v_{0}\right) \\
& \hat{Q}=e^{\partial}+\frac{2}{5}\left(u_{1}+v_{0}\right) e^{-\partial}+\frac{1}{5}\left(2 v_{0}^{\prime}-3 u_{1}^{\prime}\right) e^{-2 \partial}+ \\
& +\frac{1}{5}\left(2 u_{1}^{\prime \prime}-2 v_{0}^{\prime \prime}+\frac{4}{5} v_{0} u_{1}+u_{0}^{2}-\frac{8}{5} u_{1}^{2}-\frac{8}{5} v_{0}^{2}\right) e^{-3 \partial}+\ldots \\
c_{3}: \quad \quad & L=D^{5}+2 u_{2} D^{3}+3 u_{2}^{\prime} D^{2}+\left(2 u_{1}+3 u_{2}^{\prime \prime}\right) D+u_{1}^{\prime}+u_{2}^{\prime \prime \prime}+u_{0} D^{-1} u_{0} \\
& \hat{Q}=e^{\partial}+\frac{2}{5} u_{2} e^{-\partial}-\frac{1}{5} u_{2}^{\prime} e^{-2 \partial}+\frac{1}{5}\left(2 u_{1}+u_{2}^{\prime \prime}-\frac{8}{5} u_{2}^{2}\right) e^{-3 \partial}+\ldots
\end{aligned}
$$

The equations are for $c_{0}, c_{1}$ :

$$
\begin{aligned}
& \frac{\partial u_{2}}{\partial t_{3}}=4 u_{2}^{\prime \prime \prime}+3 u_{1}^{\prime}-\frac{12}{5} u_{2} u_{2}^{\prime} \\
& \frac{\partial u_{1}}{\partial t_{3}}=-\frac{7}{2} u_{1}^{\prime \prime \prime}+6 u_{2} u_{2}^{\prime \prime \prime}+\frac{54}{5} u_{2}^{\prime} u_{2}^{\prime \prime}+\frac{6}{5}\left(u_{2} u_{1}^{\prime}-u_{1} u_{2}^{\prime}\right)-\frac{51}{10} u_{2}^{(5)}+3 u_{0}^{\prime}
\end{aligned}
$$

for $c_{2}$ :

$$
\begin{aligned}
& 5 \frac{\partial v_{0}}{\partial t_{3}}=-v_{0}^{\prime \prime \prime}+\frac{3}{2} u_{1}^{\prime \prime \prime}-12 v_{0} v_{0}^{\prime}+6 v_{0}^{\prime} u_{1}+12 v_{0} u_{1}^{\prime} \\
& 5 \frac{\partial u_{1}}{\partial t_{3}}=6 v_{0}^{\prime \prime \prime}-4 u_{1}^{\prime \prime \prime}+15 u_{0} u_{0}^{\prime}+6 v_{0} u_{1}^{\prime}+12 v_{0}^{\prime} u_{1}-12 u_{1} u_{1}^{\prime}
\end{aligned}
$$

and for $c_{3}$ are:

$$
\begin{aligned}
& \frac{\partial u_{2}}{\partial t_{3}}=u_{2}^{\prime \prime \prime}+3 u_{1}^{\prime}-\frac{12}{5} u_{2} u_{2}^{\prime} \\
& \frac{\partial u_{1}}{\partial t_{3}}=-2 u_{1}^{\prime \prime \prime}+\frac{27}{5} u_{2}^{\prime} u_{2}^{\prime \prime}+\frac{12}{5} u_{2} u_{2}^{\prime \prime \prime}+\frac{6}{5}\left(u_{2} u_{1}^{\prime}-u_{1} u_{2}^{\prime}\right)-\frac{3}{5} u_{2}^{(5)}+3 u_{0} u_{0}^{\prime}
\end{aligned}
$$

\section{The (N,M)-KdV hierarchies}

The $(N, M)-\mathrm{KdV}$ hierarchies are defined by the pseudodifferential operator

$$
L=\partial^{N}+N \sum_{l=1}^{N-1} a_{l} \partial^{N-l-1}+N \sum_{l=1}^{M} a_{N+l-1} \frac{1}{\partial-S_{l}} \frac{1}{\partial-S_{l-1}} \ldots \frac{1}{\partial-S_{1}}, \quad N \geq 1, M \geq 0
$$


The case $(N, 0)$ coincides with the $N-\mathrm{KdV}$ case. These hierarchies were studied in [5], [6], [7], [9]. In [0] it was shown that they can be embedded in the DS construction. Now we show that this class of integrable hierarchies can be entirely embedded in the TLH. Let us see, for example, the $(2,1)$ case. The Lax operator is

$$
L=\partial^{2}+2 a_{1}+2 a_{2} \frac{1}{\partial-S}
$$

The gauge fixing gives

$$
\bar{a}_{1}=a_{1}, \quad \bar{a}_{2}=a_{2}-\frac{1}{2} a_{1}^{\prime}, \quad \bar{a}_{3}=-\frac{1}{2} a_{2}^{\prime}+\frac{1}{4} a_{1}^{\prime \prime}-\frac{1}{2} a_{1}^{2}+a_{2} S
$$

and so on. It leads, via our recipe, to the following flows

$$
\begin{aligned}
& \partial^{-1} \frac{\partial a_{1}}{\partial t_{2}}=2 a_{2}, \quad \partial^{-1} \frac{\partial a_{2}}{\partial t_{2}}=a_{2}^{\prime}+2 a_{2} S, \quad \partial^{-1} \frac{\partial S}{\partial t_{2}}=S^{2}+2 a_{1}-S, \\
& \partial^{-1} \frac{\partial a_{1}}{\partial t_{3}}=\frac{3}{2} a_{2}^{\prime}+\frac{1}{4} a_{1}^{\prime \prime}+\frac{3}{2} a_{1}^{2}+3 a_{2} S, \quad \partial^{-1} \frac{\partial a_{2}}{\partial t_{3}}=a_{2}^{\prime \prime}+3 a_{1} a_{2}+3 a_{2}^{\prime} S+3 a_{2} S^{2}
\end{aligned}
$$

and so on. These are exactly the flows of the $(2,1)-\mathrm{KdV}$ hierarchy.

\section{Comments and conclusion}

The examples we have considered in the previous section do not exhaust all possible integrable hierarchies (for an updating on this subject see [11]). However they are very numerous and they leave very little doubt that whatever scalar Lax operator (2.1), defining an integrable hierarchy, we may think of, it can be embedded in the Toda lattice hierarchy in the way we showed above. Anyhow, thus far we have not found any counterexample. Therefore our construction looks at least as general as the DS realization. The fact that we are dealing with semi-infinite matrices may suggest additional possibilities.

We also remark that the TLH, in its general formulation, may encompass several $\hat{Q}$ matrices (not only one, as in this paper). Therefore there is room for 'tensor products of integrable hierarchies in interaction'.

We end the paper by recalling that in the case of the $(1, M)-\mathrm{KdV}$ hierarchies there is a variant to the realization of section 2 . This was already pointed out in section 6.2 of [4] and, implicitly, in [5]. If one does not set $\hat{a}_{0}=0$ and replaces the first flows (2.5) in the Toda lattice flows, one gets exactly the $(1, M)$ hierarchies if the gauge fixing simply consists of setting $\hat{a}_{l}=0$ for $l>M$. It was shown in [5] that $(N, M)-\mathrm{KdV}$ hierarchies can then be extracted from the $(1, M)$ via a cascade Hamiltonian reduction. However it is not clear whether this method can be generalized to other hierarchies, and, anyhow, it does not seem to be appropriate to call it a realization of differential hierarchies, at least in the same sense this terminology has been used in this paper.

Acknowledgements. One of us (C.P.C.) would like to thank CNPq and FAPESP for financial support.

\section{References}

[1] L.A.Dickey, Soliton Equations and Hamiltonian Systems World Scientific, Singapore (1991). 
[2] V.G.Drinfeld and V.V.Sokolov, Algebras and equations of Korteweg-De Vries type, Jour.Sov.Math. 30 (1984) 1975.

[3] L.Bonora and C.S.Xiong, Multi-matrix models without continuum limit nucl.Phys. B405 (1993) 191.

[4] L.Bonora, C.P.Constantinidis and C.S.Xiong, Exact correlators of two-matrix models, SISSA 189/94/EP, hepth/9412006, to appear in Jour.Phys.Geom.

[5] L.Bonora and C.S.Xiong, Multi-field representation of the KP hierarchy and multi-matrix models, Phys.Lett.B317 (1993) 329.

[6] L.Bonora and C.S.Xiong, The $(N, M)-t h \quad K d V$ hierarchy and the associated algebras, Jour.Math.Phys. 35 (1994) 5781.

[7] L.Bonora, Q.P.Liu and C.S.Xiong, The integrable hierarchy constructed from a pair of $K d V$-type hierarchies and its associated $W$ algebra, hep-th/9408035, to be published in Comm.Math.Phys.

[8] H.Aratyn, L.A.Ferreira, J.F.Gomes and A.H.Zimerman, On two-current realization of KP hierarchy, Nucl.Phys.B402 (1993) 229.

H.Aratyn, E.Nissimov, S.Pacheva and A.H.Zimerman, Phys.Lett. 341B (1994) 19.

[9] L.A.Dickey, On the constrained KP hierarchy., Lett.Math.Phys. 34 (1995) 379; 35 (1995) 229.

[10] K.Ueno and K.Takasaki, Toda Lattice Hierarchy, Adv.Stud.Pure Math. 4 (1984) 1.

[11] Proc. W95, Marseille, July 1995, R.Grimm ed. 